\title{
Sugar-sweetened beverages intake associates with all-cause mortality independently of other dietary and lifestyle factors and obesity
}

\author{
J. J. Anderson ${ }^{1}$, S.R. Gray ${ }^{2}$, S. Iliodromity ${ }^{3}$, P. Welch ${ }^{2}$, D.F. MacKay ${ }^{1}$, C.A. Celis-Morales ${ }^{2}$, \\ D.M. Lyall ${ }^{1}$, J.M.R. Gill ${ }^{2}$, N. Sattar ${ }^{2}$ and J.P. Pell ${ }^{1}$ \\ ${ }^{1}$ Institute of Health and Wellbeing, University of Glasgow, 1 Lilybank Gardens, Glasgow, G12 8RZ, \\ ${ }^{2}$ Institute of Cardiovascular and Medical Sciences, University of Glasgow, Glasgow, G12 8TA and \\ ${ }^{3}$ School of Medicine, University of Glasgow, G12 $8 Q Q$
}

Sugar-sweetened beverages (SSB) contribute to adiposity ${ }^{(1)}$ and it is estimated that worldwide, 184,000 obesity-related deaths/year could be attributed to consumption of these drinks ${ }^{(2)}$. Recent meta-analysis suggested that consumption may be a surrogate for adverse health behaviours ${ }^{(3)}$. We used data from UK Biobank to look whether consumption of SSB, artificially-sweetened beverages (ASB) and fruit juices associates with all-cause mortality, independent of obesity and of wide range of confounders. Mortality data were obtained via linkage to the death register. Dietary information was collected via the Oxford WebQ; a web-based 24 h recall questionnaire. Univariate and multivariable Cox proportional hazard models were used to explore the associations between drinks consumption and all-cause mortality. The baseline characteristics of participants according to their intakes of sugar-sweetened beverages were compared.

After exclusion, total study population was 132,471 participants. Out of those who reported zero SSB intake/day, one SSB/day and more than one SSB/day, $1.1 \%, 1.2 \%$ and $1.3 \%$ died, respectively, over a mean follow-up period of six years. Compare to zero intake, the participants who consumed more than one SSB/day were significantly younger, men, less affluent, and less likely to be white, of lower income and less qualified. They were more likely to report higher amount of all sedentary behaviour, have higher body mass indices and report increased intake of adverse diet: eat more sugar, more fat, more total calories, more red and processed meat and lower intake of fruit and vegetable intake.

In the univariate analysis, there was a dose-response relationship between SSB consumption and all-cause mortality statistically significant for consumption of more than $1 \mathrm{SSB} /$ day. Adjustment for potential confounders did not attenuate the results (Table 1). ASB but not fruit juices were also significantly associated with increased risk of all-cause mortality.

Table 1. Cox regression analysis of the association between SSB and all-cause mortality. Unadjusted and adjusted for sex, age, ethnicity, income, qualification, physical activity, sedentary behaviour, total energy intake, body mass index, smoking, alcohol intake, intake of total sugar, fat, fruit, vegetables, and red and processed meat. Zero intake was a reference category.

\begin{tabular}{lllll}
\hline Model & HR $(95 \% \mathrm{CI})$ & HR $(95 \% \mathrm{CI})$ & P-value \\
\hline Unadjusted & $1 /$ day, $\mathrm{N}=18,888$ & P-value & $>1 /$ day, N = 8,988 & 0.015 \\
Adjusted & $1.15(1.00-1.33)$ & 0.049 & $1.27(1.05-1.53)$ & 0.007 \\
\hline
\end{tabular}

Our results show, that SSB and ASB were both associated with increased risk of mortality, independent of body weight, and this could not be explained by confounding from a wide range of socioeconomic, lifestyle and dietary factors. We propose that SSB intake could be used as a marker of adverse health choices, including adverse dietary choices and help to target individuals at risk.

1. Qi Q, Chu AY, Kang JH, Jensen MK et al. (2015) $N$ Engl J Med. 367,1387-1396.

2. Singh GM, Micha $R$, Khatibzadeh $Z$ et al. (2015) Circulation 132(8), 639-66.

3. Narain A, Kwok CS \& Mamas MA (2016) Int J Clin Pract. 70(10):791-805. 